\title{
Investigation of spatially resolved light absorption in a spark-ignition engine fueled with propane/air
}

\author{
Frank Hildenbrand, Christof Schulz, Eberhard Wagner, and Volker Sick
}

\begin{abstract}
UV absorption in the combustion phase of spark-ignition engines strongly influences laser-inducedfluorescence measurements and flame-emission techniques because of the attenuation of a laser and/or signal light. This absorption was assessed with spatial, spectral, and temporal resolutions in an optically accessible research engine. Absorption was measured along a line for different crank-angle positions throughout the combustion phase of the engine by use of spectrally resolved transmittance measurements of both broadband illumination from a deuterium lamp and emission of laser-excited hot oxygen. Evaluating the spatial patterns of absorptivity revealed that no increased absorption can be attributed to the flame-front region and that homogeneous absorption cross sections for the whole burned-gas region can be assumed. The temporal change of absorption was shown to depend on the pressure effect with only negligible changes in absorption cross sections. Results obtained from the absorption measurements are applied for spatially resolved corrections of two-dimensional laser-inducedfluorescence measurements of NO concentration fields obtained under different operating conditions. (C) 1999 Optical Society of America

OCIS codes: $120.1740,300.1030,300.2530$.
\end{abstract}

\section{Introduction}

A great variety of laser-based techniques for studying combustion processes has evolved within the past several years. ${ }^{1-4}$ Species concentrations are addressed with high spatial and temporal resolution. Furthermore, methods for measuring local temperatures have been demonstrated based on the ratio of signal intensities acquired from two-line excitation for fluorescence processes or detection of Rayleigh scattered light. All these techniques, when applied to quantitative measurements, require an exact knowledge of local intensity of the exciting laser beam and require information about the detection efficiency that depends on the transmittance of the environment under study. When laser techniques

F. Hildenbrand and C. Schulz are with the PhysikalischChemisches Institut, Universität Heidelberg, INF 253, 69120 Heidelberg, Germany. The email address for C. Schulz is christof.schulz@urz.uni-heidelberg.de. E. Wagner is with Daimler-Benz AG Forschung und Technologie 1, FT1/TV, 70546 Stuttgart, Germany. V. Sick is with the Department of Mechanical Engineering and Applied Mechanics, University of Michigan, Ann Arbor, Michigan 48109-2121. The email address for V. Sick is vsick@umich.edu.

Received 2 July 1998; revised manuscript received 21 September 1998.

0003-6935/99/091452-07\$15.00/0

(C) 1999 Optical Society of America are applied to technical combustion processes, especially under high-pressure conditions, it has been shown in engine experiments that laser beam ${ }^{5}$ and signal $^{6}$ attenuation may have a significant influence, eventually perturbing investigations of extended areas within the combustion process or leading to significant misinterpretation of signal intensities. Many of the frequently used laser techniques rely on excitation and detection in the UV spectral region, where absorption by transient combustion products such as polycyclic hydrocarbons is strong, even at small concentrations.

Providing a technique for quantifying absorption phenomena within the combustion process under study could solve these problems and enable the quantification of signal intensities. For a systematic quantification of light absorption during the combustion phase of an internal-combustion engine, two questions concerning the spatial and the temporal variabilities of absorption coefficients have to be addressed. When correcting for signal loss it has to be known whether the absorption occurs in the flamefront region only, where concentrations of transient species are expected to be maximal, or if it takes effect throughout the whole burned-gas region. Other important information concerns the temporal change of absorption. It is known from engine experiments that light attenuation increases with the development of the flame and thus with pressure. ${ }^{6,7}$ 


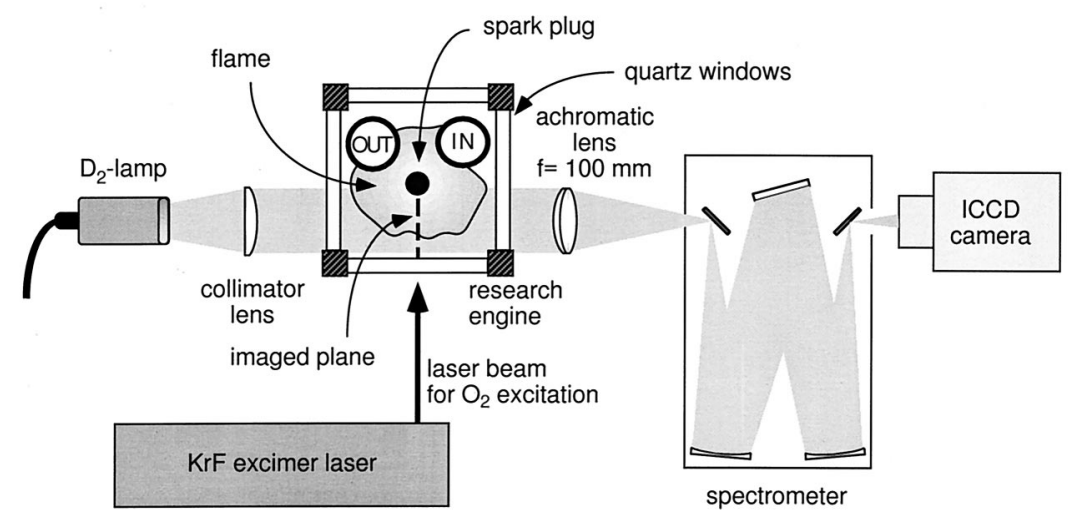

Fig. 1. Experimental setup for absorption measurements in the research engine. Two series of experiments with either a broadband $\mathrm{D}_{2}$ lamp or $\mathrm{O}_{2}$ LIF excited by a tunable $\mathrm{KrF}$ excimer laser were carried out to assess light absorption in the cylinder with spatial and spectral resolution.
It is unclear, however, if this effect depends on an increasing overall number density that is due to compression only or if the overall absorption coefficient is changing because of variations in gas composition as a result of reactions within the burned gases.

Spatially resolved measurements of the spectraltransmittance patterns, with a broadband $\mathrm{D}_{2}$ lamp as the light source, are presented here. The transmitted light was spectrally resolved with an imaging spectrometer that yielded one-dimensional spatial resolution. A research engine with optical access to the entire combustion chamber was chosen for this measurement. Extensive studies on flame development, temperature distribution, and NO concentrations were performed earlier with this engine under identical operating conditions. ${ }^{5,7-9}$ Thus the absorption data can be applied for further evaluation of the fluorescence data already available.

Besides the absorption measurements with a broadband lamp as the illumination source, which thus required an optical setup that allowed for lineof-sight measurements, an additional laser technique based on laser-induced fluorescence (LIF) of $\mathrm{O}_{2}$ was investigated. When hot bands of $\mathrm{O}_{2}$ are excited, a characteristic fluorescence-emission pattern in the UV can be generated in the plane accessible for lightsheet measurements. Since this is the plane of interest when concentration measurements are made, absorption effects can be revealed by use of the same equipment and optical setup (as depicted in Fig. 1) as those for concentration and temperature measurements simply by the tuning of the laser to an $\mathrm{O}_{2}$ resonance. When the ratio of measured and calculated emission patterns is taken, the attenuation of the signal light between the illuminated plane and the detection window can be studied with spatial and spectral resolutions.

\section{Theoretical Background}

The absorption $A$ along a line of sight (path length $d$ ) through the combustion chamber can be described according to Beer's law as long as a homogeneous absorption coefficient $\varepsilon$ can be assumed for the gas mixture with total number density $(N / V)$ :

$$
A=1-I / I_{0}=1-10^{[-(N / V) \varepsilon d]} .
$$

As is shown below, the unburned gases in the engine fueled with propane/air are transparent for the wavelengths under study. Thus absorption can be attributed to only the burned-gas region or the flame front. The flame and thus the burned-gas region are starting to evolve at the spark plug (at $x=0$ ) and are growing rather symmetrically in this flat-piston research engine. ${ }^{5}$ Therefore the absorption length $d$ varies with the distance between the middle of the combustion chamber $x=0$ and the radius of the burned-gas area $r(t)$, which in turn is time dependent as long as the flame has not reached the walls of the combustion chamber:

$$
d=c \sqrt{r(t)^{2}-x^{2}} \quad \text { for } x \leq r(t) .
$$

The factor $c$ accounts for the different geometry when broadband illumination $(c=2)$ and the fluorescence method, in which the probe light is generated in the middle of the combustion chamber $(c=1)$ and thus travels only half the way through the absorbing gases, are used.

When circular symmetry is assumed in any chosen plane of the growing flame (as proved experimentally in Refs. 5 and 9), the path length of the light through the burned-gas region can be calculated. With homogeneous absorption cross sections for the burnedgas region without enhancement in the flame-front region, the overall absorption should exhibit a maximum in the middle of the combustion chamber. On the other hand, if significant absorption occurred in the flame front, an increased absorption should be visible at the outer rim of the burned-gas area since the light path tangential to the flame front would be affected most.

\section{Experimental}

Optical access in the single-cylinder research engine was possible for the entire combustion chamber. This was achieved by a square combustion chamber enclosed by four polished quartz plates. The cross section of the combustion chamber was $75 \mathrm{~mm} \times 75$ $\mathrm{mm}$ and the piston stroke was $67 \mathrm{~mm}$. The piston was not lubricated, thus allowing for extended measurements without obscuring layers on the windows. The engine was used for extensive studies of flow 




Fig. 2. Measured pressure trace from the research engine fueled with stoichiometric propane/air mixtures in the skip-fired mode (ignition every tenth engine cycle) at 0.4-bar intake pressure. Ignition was set to $340^{\circ}$ ca.

fields, ${ }^{8}$ flame propagation, ${ }^{9}$ temperature distribution, and NO formation $5,7,10$ and is described in these references in detail. The engine was fueled with propane/air and operated at different equivalence ratios as for the fluorescence measurements ${ }^{5}$ at a speed of $1000 \mathrm{rpm}$. The engine was skip fired (i.e., only every tenth cycle was ignited), which ensured a reproducible air/fuel mixture that was free of residual combustion products that were due to an incomplete charge exchange during one cycle.

The light of a broadband $\mathrm{D}_{2}$ lamp (Heraeus, D200F) was collimated with spherical quartz lenses and directed through the combustion chamber, as depicted in Fig. 1. On the opposite side of the engine the transmitted light was focused on the entrance slit of an imaging spectrometer (Acton Research, SpectraPro-275 with correction optics and two different gratings with 300 and 1200 grooves $/ \mathrm{mm}$ ). The signal showing the spatial resolution along a line on one axis and the wavelength on the other axis was detected with an intensified CCD camera (Princeton Instruments ICCD-576 GIRB). As shown in Fig. 1, spatial resolution along a horizontal line covering one half of the combustion chamber was provided by this setup. Absorption through the whole combustion chamber was measured with a temporal resolution of $100 \mu$ s [i.e., $0.6^{\circ}$ ca (degrees crank angle) at 1000 $\mathrm{rpm}$ ] defined by the camera exposure time.

Further experiments were performed following the laser excitation of hot $\mathrm{O}_{2}$. A tunable $\mathrm{KrF}$ excimer laser (Lambda Physik LPX150) was tuned to 248.5 $\mathrm{nm}$ to excite the $P(9)$ rotational transition in the $B-X(2,7)$ band of $\mathrm{O}_{2}$, causing emission into numerous vibrational bands throughout the detected wavelength range between 220 and $325 \mathrm{~nm}$. Since the signal is emitted from only the illuminated plane, the same optical pathways apply and the same absorption phenomena are present as in the NO LIF measurements reported previously. 5,10

In all the experiments described here, ignition timing was set to $340^{\circ}$ ca $\left[20^{\circ}\right.$ before top dead center (TDC)]. The engine was operated at moderate load (0.4-bar inlet pressure), yielding peak pressures of 18 bars for stoichiometric mixtures. The pressure trace is shown in Fig. 2.

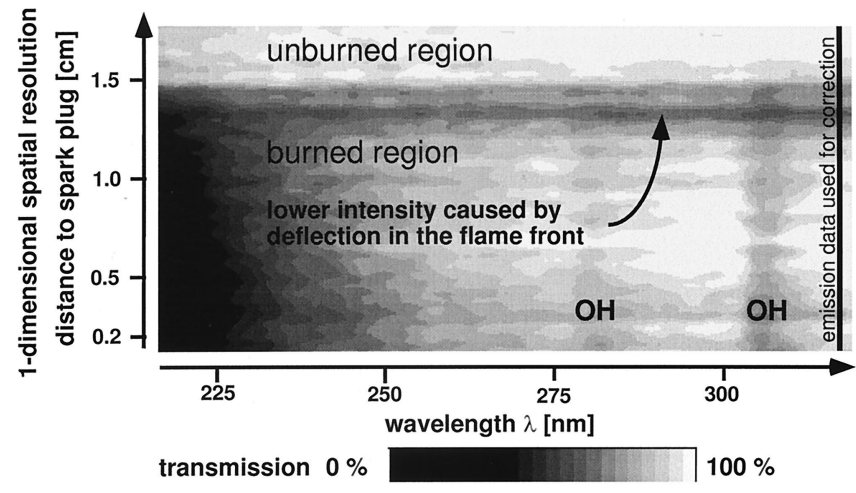

Fig. 3. Single-shot transmission spectrum obtained at $361^{\circ}$ ca at an equivalence ratio of $\phi=1.0$. The horizontal axis gives the wavelength $\lambda$ whereas the vertical axis shows the spatial position $x(x=0$ is the location of the spark plug). Dark horizontal lines at approximately $x=1.4 \mathrm{~cm}$ are due to the deflection of transmitted light close to the outer limit of the flame. Light at $\lambda=320 \mathrm{~nm}$ exhibits negligible absorption. Therefore spatial profiles at $\lambda=$ $320 \mathrm{~nm}$ were used to correct for the deflection effects. Note that the look-up table is compressed to enhance contrast.

\section{Results and Discussion}

Absorption measurements made with the optical setup described in Section 3 were performed on a single-shot basis. Besides the intensity variations that were due to absorption, further effects could be seen in the raw images. Whenever the light path was not perpendicular to the flame front, variations of the refractive index led to a deflection of the light out of the optical path of the detection system. However, unlike absorption, this deflection affected all wavelengths roughly to the same extent, causing reduced transmission that was visible as dark lines perpendicular to the spatial axis, as shown in Fig. 3. As this reduced transmission was caused by absorption, the images were corrected for this intensity decrease before interpretation. For that correction, a profile parallel to the spatial axis was taken at $\lambda=$ $320 \mathrm{~nm}$ and used to normalize the complete twodimensional spectrum obtained from the single-shot measurements. In this spectral region the absorption in the middle of the combustion chamber was reduced to $\sim 5 \%$ whereas the intensity reduction that is due to deflection persisted in the same extent as at shorter wavelengths. The deflection also positively conveniently enabled the extraction of the instantaneous flame size necessary for the calculation of the absorption path length.

For different crank-angle positions, 50 corrected single-shot spectra were averaged, yielding the spectra shown in Fig. $4\left(361^{\circ}\right.$ ca, i.e., $1^{\circ}$ ca after TDC; pressure; 11 bars; equivalence ratio; $\phi=1.0)$. Absorption, according to Eq. (1), was evaluated with transmission spectra obtained under atmosphericpressure conditions with air in the combustion chamber without operation of the engine as reference $I_{0}$. In regions where no burned gases were within the line of sight (positions greater then $15 \mathrm{~mm}$ in Fig. 3) the gas mixture was essentially transparent. At 


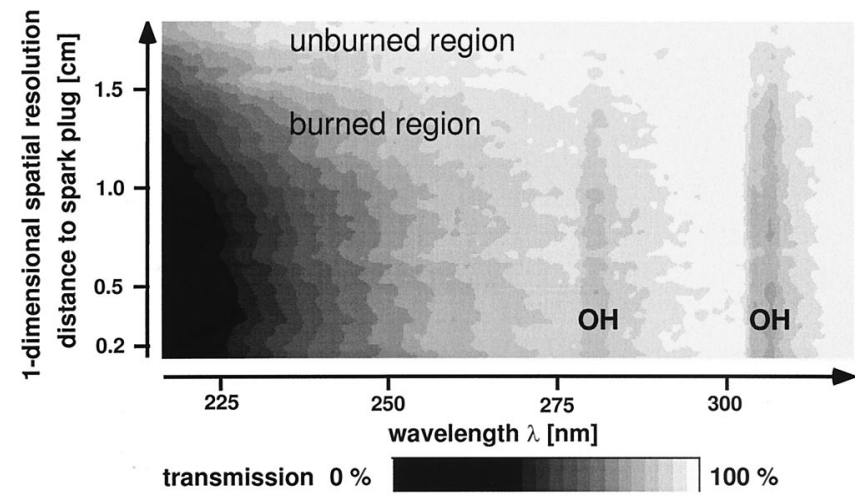

Fig. 4. Averaged, corrected transmission spectrum for the same conditions as those shown in Fig. 3.

wavelengths below $235 \mathrm{~nm}$, however, a reduced transmission was found, even in the unburned gases, resulting in a transmission of $92 \%$ at $225 \mathrm{~nm}$. In the burned-gas region the transmission was significantly reduced for wavelengths shorter than $260 \mathrm{~nm}$. Strong attenuation in the burned-gas region can be seen around 280 and $310 \mathrm{~nm}$, which can be attributed to absorption by $\mathrm{OH}$ radicals. However, no indication for narrow-band absorption by other species such as $\mathrm{NO}$ or hot $\mathrm{O}_{2}$ was seen. Therefore the absorption at wavelengths shorter than $260 \mathrm{~nm}$ is related to larger molecules such as transient unsaturated hydrocarbon species and hot ${ }^{11} \mathrm{CO}_{2}$ that show broad absorption features. A continuous increase of absorption toward the middle of the combustion chamber indicates that absorption occurs mainly within the burned gases without special contribution of transient species present in only the flame front.

Figure 5 shows a comparison of measured transmissions for 225- and 235-nm radiation across the combustion chamber with a simulation calculation

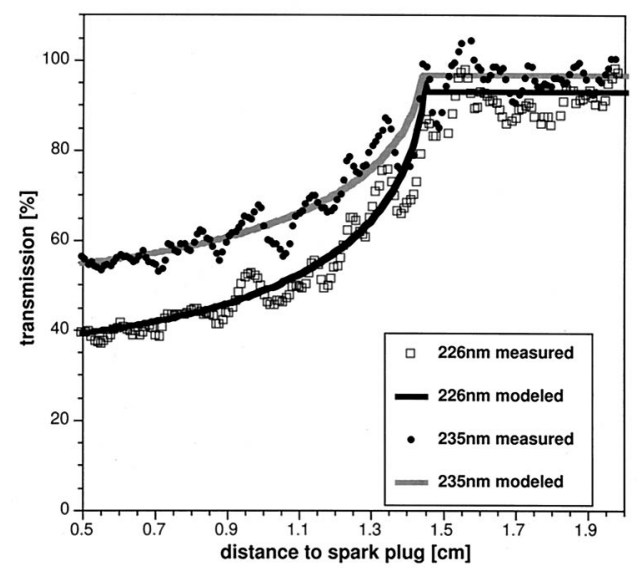

Fig. 5. Transmissions at 226 and $235 \mathrm{~nm}$ as functions of the distance to the spark plug. A comparison of measured data (as obtained from the single-shot spectra presented in Fig. 3) and simulation calculations is shown. The size of the flame was extracted from the measurements, and the absorption coefficients $\varepsilon$ (one constant value for each wavelength) were fitted to the spatially resolved transmission data.

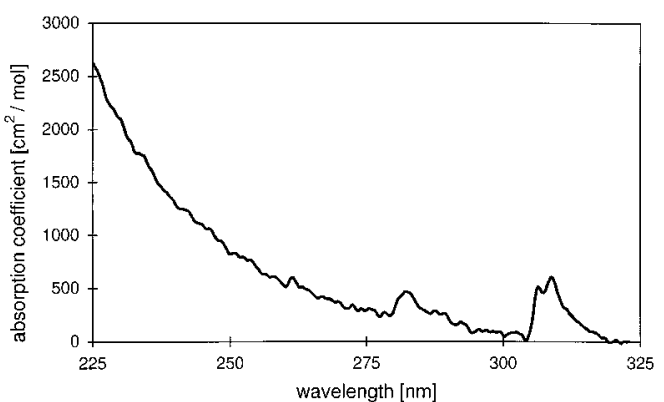

Fig. 6. Absorption coefficient $\varepsilon$ versus wavelength for the same conditions as those shown in Fig. 3. The three peaks at 261, 281 and $308 \mathrm{~nm}$ are caused by absorption due to $\mathrm{OH}$ radicals in their $(2,0),(1,0)$, and $(0,0)$ vibrational bands, respectively.

[according to Eqs. (1) and (2)] assuming spatially constant absorption coefficients $\varepsilon$ for the whole burnedgas area. Single-shot spectra were evaluated to determine the instantaneous diameter of the burnedgas region. A cylindrical symmetry of the burnedgas area was assumed to determine the different pathways of light, depending on the position within the combustion chamber. The actual absorption path length was then calculated with Eq. (2). The total number density of the burned gases $(N / V)$ was determined from the burned-gas temperature ${ }^{5}$ and the measured pressure. Equation (1) could then be computed for variable $\varepsilon$ to fit the measured spatial variation of the absorption. For the shortwavelength case $(225 \mathrm{~nm})$ an additional contribution to the absorption by the unburned gases was accounted for with an absorption coefficient of $24 \mathrm{~cm}^{2}$ / $\mathrm{mol}$, as obtained from the regions outside the flame front. The experimental data were taken from single-shot measurements. The position of the flame front at a distance of $14.5 \mathrm{~mm}$ from the middle of the combustion chamber can be clearly seen as the transmission sharply rises. Deviations from the circular flame geometry are visible as noise on the measured transmission data. The good agreement of the calculations with the experimental data indicates that the absorption is not attributed to effects within the flame front and that the assumption of homogeneous absorption throughout the whole burned-gas area is justified.

As shown in Fig. 6, the absorption coefficients are strongly dependent on the wavelength. However, their temporal change during the evolution of the flame (Fig. 7) is negligible. Furthermore it was found that $\varepsilon$ is independent of the equivalence ratio $\phi$ in the range of $\phi=0.83-1.25$ (with $\phi=1.0$ stoichiometric; $\phi>1.0$ rich flame). Therefore the absorption for individual wavelengths and any position within the combustion chamber can be calculated according to Eqs. (1) and (2) with a single absorption coefficient. Spatially resolved absorption corrections of two-dimensional LIF images can then be computed, even for images that were measured in imperfectly mixed combustion systems. 


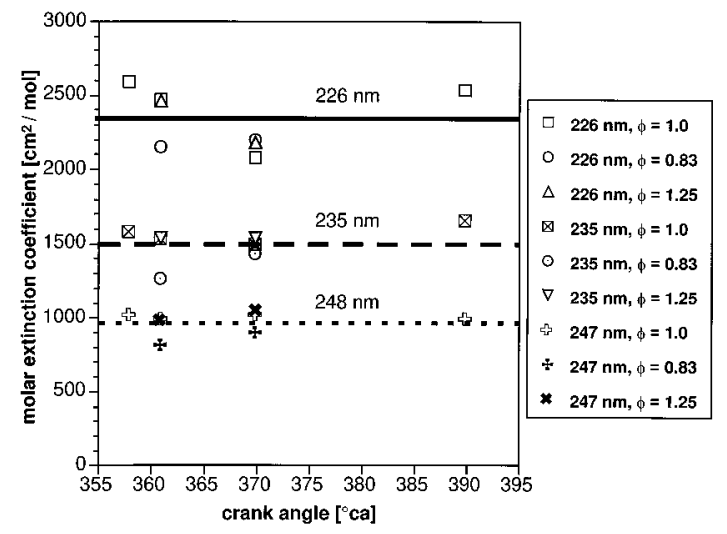

Fig. 7. Absorption coefficients $\varepsilon$ for the burned gases versus crank angle for three wavelengths relevant for NO detection. Data for different equivalence ratios $\phi$ are included.

\section{Absorption Measurements with $\mathrm{O}_{2}$ Laser-Induced Fluorescence}

The engine under study allowed full optical access, and therefore transmission could be recorded throughout the whole plane usually assessed in two-dimensional imaging measurements. However, in production engines equipped with small windows only, this technique cannot be applied. In these cases absorption between the plane illuminated during LIF measurements and the detection window can be investigated with $\mathrm{O}_{2}$ LIF. Hot $\mathrm{O}_{2}$ can easily be excited within the tuning range of a $\mathrm{KrF}$ excimer laser. When transitions in the $B-X(2,7)$ band are excited, a number of emission bands that are shifted to both shorter and longer wavelengths can be monitored. Deviations in relative signal intensities from the well-known Franck-Condon pattern can be attributed to a wavelength-dependent signal absorption. The shortwavelength region can be investigated with blueshifted $\mathrm{O}_{2}$ emission lines from transitions in the $(2,6)$ and the $(2,5)$ vibrational bands around 239 and 231 $\mathrm{nm}$, respectively. These emission bands are within the spectral range used for broadband NO detection. ${ }^{12}$ Measured LIF intensities depend on the local $\mathrm{O}_{2}$ concentration, temperature, and signal absorption. When the measured signal in the short-wavelength range is referenced to emissions in the redshifted spectral range, temperature and concentration effects cancel. Clearly, for a quantitative assessment of absorption the referencing should be done with emissions at longer wavelengths, e.g., the $(2,15)$ band at 336 $\mathrm{nm}$, where, according to Fig. 6, no absorption is expected. Unfortunately this wavelength range was not accessible with the hardware used in our LIF experiments.

Figure 8 thus displays the relative transmissions for 231 and $239 \mathrm{~nm}$ as functions of crank-angle position. The transmission from a single point in the light sheet in the center of the combustion chamber to the detection optics (see Fig. 1) is shown as an average over 100 cycle-resolved measurements. $\mathrm{O}_{2}$ was excited in the Schumann-Runge band $[B-X(2,7) P(9)$ transition] at $248.5 \mathrm{~nm}$. The transmission of each

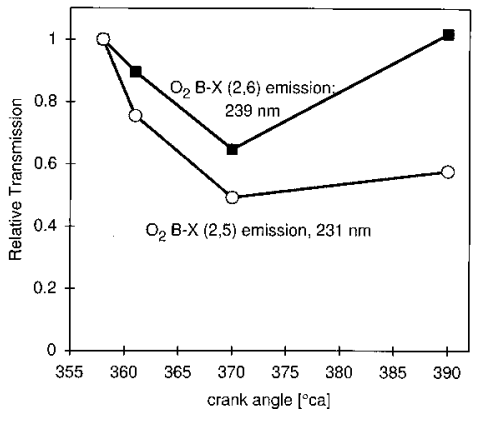

Fig. 8. Transmissions versus crank angle for 231 and $239 \mathrm{~nm}$ as obtained from the $\mathrm{O}_{2}$ LIF measurements relative to transmission at $358^{\circ}$ ca. Fluorescence intensities obtained from the $\mathrm{O}_{2}$ $B-X(2,6)$ and $(2,5)$ bands are divided by the intensities from the $(2,8)$ band at $257 \mathrm{~nm}$ to correct for $\mathrm{O}_{2}$ concentration and temperature effects.

wavelength was normalized to the measured transmission at that wavelength at $358^{\circ}$ ca. It can be seen that transmission was reduced at $\sim 370-380^{\circ}$ ca with a pronounced difference for the two bands that was due to the difference in transmission as a function of wavelength. At $390^{\circ}$ ca the transmission increased again, despite the invariant extinction coefficients as found from the broadband measurements (Fig. 7), now because of the reduced pressure in the cylinder (see Fig. 2). These findings are in good agreement with the broadband absorption measurements. Absolute absorption coefficients can be calculated from these data with Eq. (1). The absorption path length is determined with Eq. (2) with $c=$ 1 to account for the fact that, in this setup, the light travels through only half of the combustion chamber. The expected LIF intensity without signal absorption that serves as $I_{0}$ according to Eq. (1) can be calculated for any $\mathrm{O}_{2}$ emission band by use of $\mathrm{O}_{2}$ fluorescence from wavelengths longer than $310 \mathrm{~nm}$ [here $I_{\text {ref }(2,15)}$ at $336 \mathrm{~nm}$ is used as the reference]. Different transition probabilities have to be accounted for by the Einstein coefficients $A$ [not to be confused with the absorption $A$ from Eq. (1)] according to

$$
I_{0(2,5)}=I_{\text {ref }(2,15)} A_{(2,5)} / A_{(2,15)} \text {. }
$$

The transmission $T_{\lambda}$ for the wavelengths $\lambda$ is then calculated from the measured fluorescence intensity from the respective $\mathrm{O}_{2}$ fluorescence band as

$$
T_{231}=I_{(2,5)} / I_{0(2,5)} \text {. }
$$

Subscripts denote the wavelength where $I$ is measured and the vibrational bands. The use of the $(2,9)$ band emission at $266 \mathrm{~nm}$ for which the intensity is also reduced by absorption implies that the calculated value of $I_{0(2,5)}$ is underpredicted. The lack of $I_{\text {ref }(2,15)}$ prohibits the determination of $\varepsilon$ in this particular case. However, to evaluate how the $\mathrm{O}_{2} \mathrm{LIF}$ measurements compare with the broadband absorption data, Eqs. (1) and (4) are written for absorption at 231 and $239 \mathrm{~nm}$. Taking the ratio of the equa- 
tions for the two wavelengths eliminates the reference intensity $I_{\text {ref }}$ and yields

$$
\log \left(\frac{T_{231}}{T_{239}}\right)=-\frac{N}{V} d\left(\varepsilon_{231}-\varepsilon_{239}\right)
$$

With $N / V, d$, and the intensities available from the experiment, the absolute difference of the corresponding extinction coefficients can be evaluated. For the crank-angle range from 361 to $370^{\circ}$ ca the difference in the molar extinction coefficients is $550 \pm$ $180 \mathrm{~cm}^{2} / \mathrm{mol}$. This compares with $595 \pm 50 \mathrm{~cm}^{2} /$ mol obtained from the broadband measurements. For a detailed and accurate evaluation of this kind of absorption measurement over a wide range of pressures, the broadening of the $\mathrm{O}_{2}$ lines ${ }^{13}$ and the respective overlap with the laser emission bandwidth have to be included if the results are to be compared with broadband absorption measurements.

\section{Application for Corrections of Laser-Induced-Fluorescence Measurements}

Two-dimensional measurements of NO were obtained in the engine described in Ref. 5 with excitation at $248 \mathrm{~nm}$ and detection between 225 and 240 $\mathrm{nm}$. Because of the short wavelengths of the signal light, attenuation of the signal intensity had to be taken into account. The fluorescence light was detected through a side window from a vertical plane in the middle of the engine, as indicated in Fig. 1. Thus the pathway of the signal light through the combustion chamber was long $(37.5 \mathrm{~mm})$ and invariant with crank-angle position. Absolute calibration of the NO distribution measurements relied on a calibration measurement ${ }^{5,14}$ with $\mathrm{NO}$ addition in an early stage of the combustion (at $352^{\circ} \mathrm{ca}$ ). Absorption was negligible for these crank angles since the flame diameter and thus the absorption length were still small and pressure was moderate. Measurements at $352^{\circ} \mathrm{ca}$, as shown in Ref. 10 , therefore need no absorption corrections.

When measurements are performed later in the cycle or when external calibration methods are used, increasing absorption may cause underprediction of NO concentrations. Spatially resolved absorption corrections must include different path lengths [according to Eq. (2)] for LIF signals through the burnedgas region. A comparison of corrected and uncorrected images of averaged NO concentration distributions is shown in Fig. 9 for both stoichiometric and fuel-rich operations at $355^{\circ} \mathrm{ca}$. Corresponding profiles taken along the lines indicated in Fig. 8 show the magnitude of the absorption correction. In the middle of the combustion chamber, correction enhances measured NO concentrations by $35 \%$. At later crank angles connected to higher pressures and larger flame diameters, correction can rise up to a factor of 2 (at $361^{\circ} \mathrm{ca}$ ), as calculated from the absorption coefficients.

Although this paper addresses the fundamental features of signal absorption, an extensive discussion


Fig. 9. Averaged NO concentration fields obtained from twodimensional LIF measurements ${ }^{5}$ with and without corrections for absorption of the signal light. Profiles along the lines indicated in the images are given in the graph at the bottom.

of measured NO distributions for a variety of engine operating conditions is in progress.

\section{Conclusions}

UV absorption observed in a spark-ignition (SI) engine during the combustion phase was quantified with spatial, spectral, and temporal resolutions. Both a broadband $\mathrm{D}_{2}$ lamp and $\mathrm{O}_{2}$ LIF were used for assessing the absorption effects that have been found in extensive laser diagnostic measurements carried out earlier in the same engine under identical operating conditions.

From comparisons between measurements and calculations it was found that (1) absorption occurs throughout the whole burned-gas area without further increase within the flame front, and (2) absorption coefficients are invariant with time and equivalence ratio (confirmed for propane/air, $\phi=$ 0.83-1.25). Thus an increasing absorption during evolution of the flame can be attributed to the pressure (total number density) rise only. These findings allow for corrections of absorption phenomena when the flame development and thus the path length of the signal light through the burned-gas area are known.

The effects of absorption on measured NO concentration fields obtained in the same engine were presented. Absorption was shown to be severe in the burned gases in a SI engine that has the potential of 
perturbing fluorescence techniques. Likewise, flameluminosity measurements, ${ }^{15}$ such as those frequently used for concentration and temperature investigations, will be affected.

The present study focuses on the engine fueled with premixed propane/air. Further studies have to be carried out with different fuels and stratifiedload SI engines. However, no major changes are expected for the burned-gas composition as long as the fuel is completely burned. Under fuel-rich conditions, in sooting environments, or under conditions in which flame quenching occurs, significant changes in absorption phenomena can be expected.

The detection of signal light through side windows is not desirable when short-wavelength detection around TDC is required. Detection through windows in the piston ${ }^{16}$ or the cylinder head ${ }^{17}$ minimizes the absorption path length since the illuminated plane is close to the detection window.

The authors thank K. Koyanagi (Daimler-Benz) and H. Krämer [Physikalisch-Chemisches Institut (PCI), University of Heidelberg] for assistance during the measurement and J. Wolfrum (PCI) for his continuous encouragement of this research. This study has been funded by the Bundesministerium für Bildung, Forschung und Technologie under contract 13N6283 (PCI, University of Heidelberg).

\section{References}

1. A. C. Eckbreth, Laser Diagnostics for Combustion, Temperature and Species (Gordon \& Breach, Amsterdam, 1996).

2. K. Kohse-Höinghaus, "Laser techniques for the quantitative detection of reactive intermediates in combustion systems," Prog. Energ. Combust. Sci. 20, 203-247 (1994).

3. E. W. Rothe and P. Andresen, "Application of tunable excimer lasers to combustion diagnostics: a review," Appl. Opt. 36, 3971-4033 (1997).

4. H. Zhao and N. Ladommatos, "Optical diagnostics for incylinder mixture formation measurements in IC engines," Prog. Energ. Combust. Sci. 24, 297-336 (1998).

5. C. Schulz, V. Sick, J. Wolfrum, V. Drewes, M. Zahn, and R. Maly "Quantitative 2D single-shot imaging of NO concentrations and temperatures in a transparent SI engine," in Proceedings of the Twenty-Sixth International Symposium on Combustion (The Combustion Institute, Pittsburgh, Pa., 1996), pp. 2597-2604.

6. M. Knapp, A. Luczak, H. Schlüter, V. Beushausen, W.
Hentschel, and P. Andresen, "Crank-angle-resolved laserinduced fluorescence imaging of NO in a spark-ignition engine at $248 \mathrm{~nm}$ and correlations to flame front propagation and pressure release," Appl. Opt. 35, 4009-4017 (1996).

7. A. Bräumer, V. Sick, J. Wolfrum, V. Drewes, R. R. Maly, and M. Zahn, "Quantitative two-dimensional measurements of nitric oxide and temperature in a transparent SI engine," in 1995 SAE Fuels and Lubricants Meeting (Society of Automotive Engineers, Warrendale, Pa., 1995), paper 952462.

8. W. Stolz, J. Köhler, W. Lawrenz, F. Meier, W. H. Bloss, R. R. Maly, R. Herweg, and M. Zahn, "Cycle-resolved flow field measurements using a PIV movie technique in an SI engine," in 1992 SAE Congress and Exposition (Society of Automotive Engineers, Warrendale, Pa., 1992), paper 922354.

9. R. R. Maly and M. Zahn, "Engine and fuel interactions," Final Rep. Contract JOU2-CT92-0081 (The Comission of the European Communities, Brussels, Belgium, 1995).

10. C. Schulz, J. Wolfrum, and V. Sick, "Comparative study of experimental and numerical NO profiles in SI combustion," in Proceedings of the Twenty-Seventh International Symposium on Combustion (The Combustion Institute, Pittsburgh, Pa., 1998).

11. R. J. Jensen, R. D. Guettler, and J. L. Lyman, "The ultraviolet absorption spectrum of hot carbon dioxide," Chem. Phys. Lett. 277, 356-360 (1997).

12. C. Schulz, V. Sick, J. Heinze, and W. Stricker, "Laserinduced fluorescence detection of nitric oxide in highpressure flames with $A-X(0,2)$ excitation," Appl. Opt. 36, 3227-3232 (1997).

13. V. Sick, M. Decker, J. Heinze, and W. Stricker, "Collisional processes in the $B$ state of $\mathrm{O}_{2}$," Chem. Phys. Lett. 249, 335-340 (1996).

14. C. Schulz, V. Sick, and U. Meier, "Quantification of NO $A-X(0,2)$ laser-induced fluorescence: investigation of calibration and collisional influences in high-pressure flames," Submitted to Appl. Opt. (1998).

15. P. Witze, "In-cylinder diagnostics for production spark ignition engines," in Unsteady Combustion, F. Culick, M. V. Heitor, and J. H. Whitelaw, eds., Vol. 306 of NATO ASI Series (Kluwer, Dordrecht, The Netherlands, 1996).

16. F. Hildenbrand, C. Schulz, V. Sick, G. Josefsson, I. Magnusson, Ö. Andersson, and M. Aldén, "Laser spectroscopic investigation of flow fields and NO-formation in a realistic SI engine," in 1998 SAE Congress and Exposition (Society of Automotive Engineers, Warrendale, Pa., 1998), paper 980148.

17. J. E. Dec and R. E. Canaan, "PLIF imaging of NO formation in a DI diesel engine," in 1998 SAE Congress and Exposition (Society of Automotive Engineers, Warrendale, Pa., 1998), paper 980147. 